\title{
Nanomedicines in the treatment of brain tumors
}

\author{
Funmilola A Fisusi ${ }^{1,2}$, Andreas G Schätzlein ${ }^{1,3}$ \& ljeoma F Uchegbu*,1,3 \\ ${ }^{1}$ UCL School of Pharmacy, 29-39 Brunswick Square, London, WC1N 1AX, UK \\ ${ }^{2}$ Drug Research \& Production Unit, Faculty of Pharmacy, Obafemi Awolowo University, lle-lfe, Nigeria \\ ${ }^{3}$ Nanomerics Ltd., New Bridge Street House, 30-34 New Bridge Street, London, EC4V 6BJ, UK \\ * Author for correspondence: ucnvifu@ucl.ac.uk
}

\section{${ }^{6} \mathrm{~A}$ major contribution to the poor survival rates is the insufficient transport of therapeutic molecules across the blood-brain barrier"}

First draft submitted: 15 December 2017; Accepted for publication: 19 December 2017; Published online: 29 January 2018

Keywords: brain tumor $\bullet$ doxorubicin $\bullet$ glioblastoma $\bullet$ nanomedicine $\bullet$ nanoparticle $\bullet$ paclitaxel $\bullet$ theranostic

\section{Brain metastases \& primary CNS tumors}

Brain metastases are the most frequently occurring neurologic complications of cancer in adults, with $9-17 \%$ of all cancers resulting in brain metastasis and brain metastasis occurring in 8-14 per 100,000 in the general population [1]. Primary brain tumors, on the other hand, are relatively rare, and comprise about $1.4 \%$ of cancers [2]. Brain metastases are associated with median survival times of about 3-25 months [3], and a 5-year survival rate of $1.8 \%$ [4]. Treatment modalities employed for brain metastases include: surgical resection, whole brain radiation therapy, radiosurgery and chemotherapy [5]. The choice of treatment would usually be based on several considerations. These include: histopathology of the primary tumor, status of systemic disease, patient's performance status (general well being and lifestyle activity level), age of the patient, number and sites and precise location of the brain metastases (such as proximity to sites of vital brain function), coexisting morbidities and symptoms [2,5].

Glioblastoma multiforme (WHO Classification astrocytoma Grade IV), a metastatic primary brain tumor, accounts for $12-15 \%$ of all brain tumors [6] and is the most common primary brain tumor in adults [7]. Glioblastoma is an aggressive metastatic astrocytoma with a median survival of 14 months and $<5 \%$ of patients survive for 3 years [8]. This tumor is difficult to diagnose early as the tumor is usually asymptomatic or presents with symptoms which are difficult to associate with GBM, for example, symptoms associated with a high intracranial pressure (headaches, nausea, vomiting and cognitive impairment) [9]. A major contribution to the poor survival rates is the insufficient transport of therapeutic molecules across the blood-brain barrier (BBB) [10]. The current standard of care comprises surgical resection to the maximum possible extent, followed by concurrent radio chemotherapy and adjuvant chemotherapy with temozolomide [2]. This treatment regimen became the standard of care for newly diagnosed glioblastoma patients after the results of the 2004 European Organisation for Research and Treatment of Cancer 26981-22981/National Cancer Institute of Canada Clinical Trials Group CE3 randomized Phase III trial demonstrated a $20.7 \%$ improvement in the median survival as well as $27.2 \%$ 2-year survival rates in glioblastoma patients, who had received postsurgical concomitant and adjuvant temozolomide (known as the Stupp regimen) compared with $10.9 \%$ 2-year survival rates with postsurgical radiotherapy alone [11]. For recurrent glioblastoma on the other hand, there is currently no standard treatment regimen [12], and thus patients frequently receive investigational agents in clinical trials [13].

\section{The blood-brain barrier}

The treatment of brain tumors (or more generally, CNS tumors) is particularly challenging, mainly because of their intracranial location [14]. Intracranial tumors are effectively 'shielded' from the effects of most systemically administered cytotoxic agents. The brain parenchyma and most (but not all) intracranial tumors are protected by the intact $\mathrm{BBB}$, which maintains the brain microenvironment by serving as a physical and metabolic barrier 
regulating the access of molecules to the brain [15]. The physical barrier is formed by the tight junctions between the adjacent endothelial cells (which prevent blood-borne substances from crossing into the brain parenchyma), a lack of capillary fenestrations, very low pinocytotic activity and the metabolic barrier is formed by degradative enzymes, specialized transport receptors and endothelial cell efflux pumps [15].

\section{Other brain tumor treatment barriers}

Another barrier thought to restrict access of systemically administered therapeutic agents to tumor cells is the brain tumor-cell barrier (BTB; a barrier caused by the efflux activity of tumor cells) [16]. Other challenges associated with effective brain tumor treatment are: dose-limiting toxicity, mainly myelosuppression and tumor resistance to alkylating agents; the latter mediated mainly by MGMT [14].

\section{Passive targeting with nanoparticles}

Nanoparticles have been used to passively target drugs to intracranial tumors, on intravenous injection, in order to enable delivery of therapeutics across the BBB to the brain, as there is evidence that nanoparticles are able to preferentially accumulate drug at tumor sites, when compared with the administration of drugs in solution [17]. Generally nanoparticles may be engineered to: enable tissue or organ-specific transport of their drug payload; or enable the delivery of hydrophobic and metabolically labile drugs $[18,19]$. Thus, nanoparticles are an interesting platform to consider in drug development for brain tumor indications [19].

Intravenously administered nanoparticles for delivery of therapeutic agents to brain tumors may theoretically exploit the enhanced permeability and retention effect, whereby particles extravasate through a leaky tumor vasculature and achieve closer proximity to the tumor cells [20]. However, for the enhanced permeability and retention effect to be operational, the $\mathrm{BBB}$ must be compromised at the site of the intracranial tumor and while the breakdown of the BBB is diagnostic of a high grade glioma [21] most tumors are associated with an intact BBB [22] and direct evidence of nanoparticle accumulation within intracranial tumor cells is difficult to find. Early activity in this area focused on the delivery of P-gp efflux pump substrates to the brain in an attempt to circumvent the brain tumor-cell barrier. The P-gp substrate [23], doxorubicin, when intravenously injected in poly(butylcyanoacrylate) nanoparticles, resulted in increased tumor tissue accumulation of the drug, in a C6 glioma rat model, when compared with healthy tissue and an attendant improvement in tumoricidal activity was observed with these nanoparticles when compared with the drug in solution [24]. Additionally, the formulation was also found to be less cardiotoxic. This provides indirect evidence that nanoparticles are able to take advantage of a variation in the BBB at the tumor site.

As well as the cyanoacrylates, other polymers have also demonstrated the tumor tissue drug accumulation phenomenon on intravenous injection. Doxorubicin loaded on to poloxamer 188-coated poly-(lactic acid-coglycolic acid) (PLGA) nanoparticles in a rat glioblastoma 101/8 model resulted in superior tumoricidal activity, through the intravenous route, when compared with the drug in solution [25].

Nanomedicines may also consist of more than one therapeutic for the treatment of brain tumors. For example, chitosan surface-modified PLGA nanoparticles loaded with carmustine along with $\mathrm{O}^{6}$-benzylguanine (which depletes MGMT, thus improving the therapeutic efficacy of carmustine). On intravenous injection, this nanoparticle formulation yielded superior survival outcomes in F98 glioma-bearing rats compared with the administration of the two drugs separately in solution or to the nanoparticle containing carmustine alone [26].

Nanoparticles may also work by simply increasing plasma exposure, which in turn increases brain exposure, while minimizing exposure to areas of potential toxicity [27]. We have shown that lomustine loaded on to GCPQ (N-palmitoyl-N-monomethyl-N,N-dimethyl-N,N,N-trimethyl-6-O-glycolchitosan) nanoparticles resulted in increased plasma and brain exposure, reduced liver and bone exposure and ultimately increased tumoricidal activity (survival and tumor size) in a U87MG intracranial tumor model, without increasing myelosuppression [27].

\section{Active targeting with nanoparticles}

Active targeting involves the use of carriers bearing various surface ligands to achieve either transport across an intact $\mathrm{BBB}$, or alternatively, cell uptake following extravasation across a leaky BBB [28].

Various across BBB transporters such as TfR [29] and GLUT have been exploited for the transport of drugs across an intact $\mathrm{BBB}$ [30]. Tf - c[RGDfK] paclitaxel micelles have been prepared and injected intravenously to a U87MG mouse model, with Tf included to enable across $\mathrm{BBB}$ transport while $\mathrm{c}[\mathrm{RGDfK}]$ was included to enable uptake by tumor cells [29]. This resulted in drug accumulation in the brain and a superior anti-glioma effect compared with 


\begin{tabular}{|c|c|c|c|c|c|}
\hline Study number & Nanoparticle type & Drug & Indication & Study Phase & Ref. \\
\hline NCT02340156 & Cationic liposomes & $\begin{array}{l}\text { Liposomes encapsulated } p 53 \\
\text { cDNA in combination with oral } \\
\text { temozolomide }\end{array}$ & Recurrent glioblastoma & Phase II & [35] \\
\hline NCT02820454 & Polymer-gadolinium chelates & $\begin{array}{l}\text { AGuIX (polysiloxane } \\
\text { gadolinium-chelates-based } \\
\text { nanoparticles) concurrently with } \\
\text { whole brain radiation }\end{array}$ & Brain metastases & Phase I & [35] \\
\hline NCT01266096 & Silica & $\begin{array}{l}\text { 124I-cRGDY-PEG-dots for PET } \\
\text { scan }\end{array}$ & $\begin{array}{l}\text { Newly diagnosed or } \\
\text { recurrent metastatic } \\
\text { melanoma, malignant } \\
\text { brain tumors }\end{array}$ & Microdosing study & [35] \\
\hline NCT03020017 & Gold & NU-0129 & $\begin{array}{l}\text { Gliosarcoma, recurrent } \\
\text { glioblastoma }\end{array}$ & Early Phase I & [35] \\
\hline NCT00734682 & Liposome & CPT-11 & $\begin{array}{l}\text { Recurrent high-grade } \\
\text { gliomas }\end{array}$ & Phase I & [35] \\
\hline
\end{tabular}

the commercial formulation, Taxol [29]. Others have utilized the T7 peptide (HAIYPRH) to target the endothelial cell TfR and achieve across BBB transport [31]. T7 peptide-modified core-shell nanoparticles (T7-LPC/siRNA) have been shown, on intravenous administration, to accumulate anti-EGFR siRNA in intracranial tumor tissue, downregulate EGFR and increase survival rates in a U87MG mouse tumor model, when compared with plain nanoparticles [31]. While the T7 peptide appears to achieve delivery across the BBB [31], efforts to improve cell uptake, once extravasation has taken place, have involved the use of dual targeting strategies, in which transport across the BBB is combined with a ligand promoting tumor cell uptake [32]. Intravenously administered dualtargeted doxorubicin liposomes comprising the TAT peptide for cellular uptake and the T7 peptide for across BBB transport resulted in increased delivery of doxorubicin to the brain glioma tissue in a C6 glioma mouse model and reduced delivery to the heart, which is relevant for the cardiotoxic [33] drug doxorubicin [32].

Utilization of GLUT to cross the BBB has been achieved by using 2-deoxy-D-glucose-modified poly(ethylene glycol)-co-poly(trimethylene carbonate) paclitaxel nanoparticles [30]. The 2-deoxy-D-glucose moiety was correlated with drug accumulation in the brain and these glucose-decorated nanoparticles produced superior survival in an RG2 mouse glioma model, when compared with plain nanoparticles and Taxol [30].

While there is good preclinical evidence showing the efficacy of nanoparticles in rodent models of intracranial tumors, clinical evidence on the use of nanoparticles is harder to locate. There are some reports of clinical trials in brain tumor patients with passively targeted nanoparticles (Table 1): for example, NCT02340156, NCT02820454, NCT01266096, NCT03020017, NCT00734682 [34,35]; however, the efficacy of this nanoparticle approach in the clinic has not yet been reported.

\section{Theranostics}

Imaging agents and drugs transported by a single nanoparticle are another area of innovation that has been applied to the treatment of experimental brain tumors and these are known as theranostics $[17,36]$. Intravenously administered polymeric nanoparticles loaded with smaller iron oxide nanoparticles (for MRI), surface decorated with a tumor vasculature targeting F3 peptide (a 31-amino acid sequence of the amino-terminal fragment of human high-mobility group protein 2) and encapsulating photofrin for photodynamic therapy (PDT), were accumulated within the intracranial tumor in a 9L glioma rat model, following intravenous administration, as visualized using MRI [37]. This theranostic improved survival rates in this model following PDT when compared with plain nanoparticles in combination with PDT or photofrin alone in combination with PDT. Iron oxide as an MRI imaging agent is the contrast enhancement agent of choice with a number of theranostics. The combination of a tumor-homing peptide (CGKRK), which targets the tumor endothelial and tumor cells and specifically their mitochondria with a proapoptotic peptide $(\mathrm{D}[\mathrm{KLAKLAK}] 2)$ as the drug, when coupled to elongated iron oxide nanoparticles (nanoworms), as the MRI contrast agent, has been shown to accumulate these targeted nanoworms in the tumor tissue following intravenous injection [38]. The targeted nanoworms were significantly more effective than nontargeted nanoworms in a lentiviral (H-RasV12-shp53)-induced mouse brain tumor model.

An alternative method of labeling nanoparticles for imaging in a theranostic platform involves the use of porphyrin for near infrared imaging and as such $30 \mathrm{~nm}$ porphyrin-lipid ApoE3 lipid nanoparticles (pyE-LNs) with 
intrinsic imaging properties via the porphyrin lipid have been studied [39]. Across BBB delivery and tumor cell uptake properties were achieved using $\mathrm{ApoE}$ as $\mathrm{ApoE}$ is taken up by the low-density lipoprotein receptor on brain endothelial cells and tumor cells, where in the latter case, the low-density lipoprotein receptor is upregulated [39]. After intravenous administration to a U87 Green Fluorescent Protein mouse model, the particles were found to accumulate within brain tumor tissue.

These image-competent nanotherapeutics may prove interesting in the treatment of diffuse brain metastasis in multiple brain regions.

\section{Conclusion}

Glioblastoma and brain metastasis are still areas of unmet medical need and several nanoparticle formulations are showing promise in glioblastoma rodent models of the disease with a few even transitioning to clinical testing. The leaky vasculature in brain tumors has been exploited to concentrate drug-laden nanoparticles at the tumor site, following intravenous injection. Additionally, various across BBB transport and cell uptake ligands have been employed within a single nanoparticle to enable drug to be concentrated in tumor cells in the presence of an intact BBB, following intravenous injection. These combined systems are known as dual-targeting systems. Recent studies have introduced MRI and near infrared imaging to the administration of drug-loaded nanoparticles, enabling targeting to be imaged with these new theranostics. Tfr has been widely exploited for across BBB transport, in these experimental studies, and a number of cell uptake ligands employed in the dual-targeting approaches. It remains to be seen if the promising rodent data are indeed translated to approved clinical therapies and attention will need to be turned to the issue of manufacturability if the ligand-targeting systems are to transition into clinical products.

\section{Financial \& competing interests disclosure}

The authors have no relevant affiliations or financial involvement with any organization or entity with a financial interest in or financial conflict with the subject matter or materials discussed in the manuscript. This includes employment, consultancies, honoraria, stock ownership or options, expert testimony, grants or patents received or pending, or royalties.

No writing assistance was utilized in the production of this manuscript.

\section{References}

Papers of special note have been highlighted as: $\bullet$ of interest

1 Nayak L, Lee EQ, Wen PY. Epidemiology of brain metastases. Curr. Oncol. Rep. 14(1), 48-54 (2012).

2 Kemmerer E, Shah S. Update on radiotherapy for central nervous system malignancies. Surg. Oncol. Clin. N. Am. 26(3), 347-355 (2017).

3 Sperduto PW, Kased N, Roberge D et al. Summary report on the graded prognostic assessment: an accurate and facile diagnosis-specific tool to estimate survival for patients with brain metastases. J. Clin. Oncol. 30(4), 419-425 (2012).

4 Lutterbach J, Bartelt S, Ostertag C. Long-term survival in patients with brain metastases. J. Cancer Res. Clin. Oncol. 128(8), 417-425 (2002).

5 Hardesty DA, Nakaji P. The current and future treatment of brain metastases. Front. Surg.doi:10.3389/fsurg.2016.00030 (2016) (Epub ahead of print).

6 Iacob G, Dinca EB. Current data and strategy in glioblastoma multiforme. J. Med. Life 2(4), 386-393 (2009).

7 Furnari FB, Fenton T, Bachoo RM et al. Malignant astrocytic glioma: genetics, biology, and paths to treatment. Genes Dev. 21(21), 2683-2710 (2007).

8 Krex D, Klink B, Hartmann C et al. Long-term survival with glioblastoma multiforme. Brain 130(Pt 10), 2596-2606 (2007).

9 Urbanska K, Sokolowska J, Szmidt M, Sysa P. Glioblastoma multiforme - an overview. Contemp Oncol. (Pozn.) 18(5), 307-312 (2014).

10 Agarwal S, Manchanda P, Vogelbaum MA, Ohlfest JR, Elmquist WF. Function of the blood-brain barrier and restriction of drug delivery to invasive glioma cells: findings in an orthotopic rat xenograft model of glioma. Drug Metab. Dispos. 41(1), 33-39 (2013).

11 Stupp R, Mason WP, van den Bent MJ et al. Radiotherapy plus concomitant and adjuvant temozolomide for glioblastoma. N. Engl. J. Med. 352(10), 987-996 (2005).

12 Weller M, Cloughesy T, Perry JR, Wick W. Standards of care for treatment of recurrent glioblastoma - are we there yet? Neuro-Oncology 15(1), 4-27 (2013).

13 Wong ET, Hess KR, Gleason MJ et al. Outcomes and prognostic factors in recurrent glioma patients enrolled onto Phase II clinical trials. J.Clin. Oncol. 17(8), 2572-2578 (1999).

14 Raizer J. Issues in developing drugs for primary brain tumors: barriers and toxicities. Toxicol. Pathol. 39(1), 152-157 (2011). 
15 Abbott NJ, Ronnback L, Hansson E. Astrocyte-endothelial interactions at the blood-brain barrier. Nat. Rev. Neurosci. 7(1), 41-53 (2006).

16 Agarwal S, Sane R, Oberoi R, Ohlfest JR, Elmquist WF. Delivery of molecularly targeted therapy to malignant glioma, a disease of the whole brain. Expert Rev. Mol. Med. 13, e17 (2011).

17 Davis ME, Chen Z, Shin DM. Nanoparticle therapeutics: an emerging treatment modality for cancer. Nat. Rev. Drug Discov. 7(9), 771-782 (2008).

18 Drbohlavova J, Chomoucka J, Adam V et al. Nanocarriers for anticancer drugs - new trends in nanomedicine. Curr. Drug Metab. 14(5), 547-564 (2013).

19 Bhojani MS, Van Dort M, Rehemtulla A, Ross BD. Targeted imaging and therapy of brain cancer using theranostic nanoparticles. Mol. Pharmaceut. 7(6), 1921-1929 (2010).

20 Maeda H, Wu J, Sawa T, Matsumura Y, Hori K. Tumour vascular permeability and the EPR effect in macromolecular therapeutics: a review. J. Controlled Release, 65, 271-284 (2000).

21 Dhermain FG, Hau P, Lanfermann H, Jacobs AH, van den Bent MJ. Advanced MRI and PET imaging for assessment of treatment response in patients with gliomas. Lancet Neurol. 9(9), 906-920 (2010).

22 van Tellingen O, Yetkin-Arik B, de Gooijer MC, Wesseling P, Wurdinger T, de Vries HE. Overcoming the blood-brain tumor barrier for effective glioblastoma treatment. Drug Resist. Updat. 19, 1-12 (2015).

23 Uchegbu IF, Double JA, Kelland LR, Turton JA, Florence AT. The activity of doxorubicin niosomes against an ovarian cancer cell line and three in vivo mouse tumour models. J. Drug Target 3(5), 399-409 (1996).

24 Zhang Y, Yu J, Zhang L, Cai J, Cai D, Lv C. Enhanced anti-tumor effects of doxorubicin on glioma by entrapping in polybutylcyanoacrylate nanoparticles. Tumor Biol. 37(2), 2703-2708 (2016).

25 Wohlfart $S$, Khalansky AS, Gelperina $S$ et al. Efficient chemotherapy of rat glioblastoma using doxorubicin-loaded PLGA nanoparticles with different stabilizers. PLOS ONE 6(5), e19121 (2011).

26 Qian L, Zheng J, Wang K et al. Cationic core-shell nanoparticles with carmustine contained within O(6)-benzylguanine shell for glioma therapy. Biomaterials 34(35), 8968-8978 (2013).

27 Fisusi FA, Siew A, Chooi KW et al. Lomustine nanoparticles enable both bone marrow sparing and high brain drug levels - a strategy for brain cancer treatments. Pharmaceut. Res. 33(5), 1289-1303 (2016).

28 Karim R, Palazzo C, Evrard B, Piel G. Nanocarriers for the treatment of glioblastoma multiforme: current state-of-the-art. J. Control. Release 227, 23-37 (2016).

29 Zhang P, Hu L, Yin Q, Feng L, Li Y. Transferrin-modified c[RGDfK]-paclitaxel loaded hybrid micelle for sequential blood-brain barrier penetration and glioma targeting therapy. Mol. Pharmaceut. 9(6), 1590-1598 (2012).

30 Jiang X, Xin H, Ren Q et al. Nanoparticles of 2-deoxy-D-glucose functionalized poly(ethylene glycol)-co-poly(trimethylene carbonate) for dual-targeted drug delivery in glioma treatment. Biomaterials 35(1), 518-529 (2014).

31 Wei L, Guo XY, Yang T, Yu MZ, Chen DW, Wang JC. Brain tumor-targeted therapy by systemic delivery of siRNA with transferrin receptor-mediated core-shell nanoparticles. Int. J. Pharmaceut. 510(1), 394-405 (2016).

32 Zong T, Mei L, Gao H et al. Enhanced glioma targeting and penetration by dual-targeting liposome co-modified with T7 and TAT. J. Pharmaceut. Sci. 103(12), 3891-3901 (2014).

33 Octavia Y, Tocchetti CG, Gabrielson KL, Janssens S, Crijns HJ, Moens AL. Doxorubicin-induced cardiomyopathy: from molecular mechanisms to therapeutic strategies. J. Mol. Cell Cardiol. 52(6), 1213-1225 (2012).

34 Anselmo AC, Mitragotri S. Nanoparticles in the clinic. Bioeng. Translat. Med. 1(1), 10-29 (2016).

35 Medicine USlo. https://clinicaltrials.gov/

36 Meyers JD, Doane T, Burda C, Basilion JP. Nanoparticles for imaging and treating brain cancer. Nanomedicine (Lond.) 8(1), 123-143 (2013).

37 Reddy GR, Bhojani MS, McConville P et al. Vascular targeted nanoparticles for imaging and treatment of brain tumors. Clin. Cancer Res. 12(22), 6677-6686 (2006).

38 Agemy L, Friedmann-Morvinski D, Kotamraju VR et al. Targeted nanoparticle enhanced proapoptotic peptide as potential therapy for glioblastoma. Proc. Natl Acad. Sci. USA 108(42), 17450-17455 (2011).

39 Rajora MA, Ding L, Valic M et al. Tailored theranostic apolipoprotein E3 porphyrin-lipid nanoparticles target glioblastoma. Chem. Sci. 8(8), 5371-5384 (2017).

- Electronic supplementary information available. 
